\title{
Gas Dynamics and Active Phenomena in Galactic Nuclei: M100 and M94
}

\author{
Kazushi Sakamoto 1 \\ Nobeyama Radio Observatory, Minamisaku, Nagano, 384-13, Japan \\ Takeo Minezaki ${ }^{1}$ \\ National Astronomical Observatory, Mitaka, Tokyo, 181, Japan \\ Keiichi Wada \\ Center for Information Processing Education, Hokkaido University, \\ Sapporo, 060, Japan \\ Sachiko Okumura \\ Nobeyama Radio Observatory, Minamisaku, Nagano, 384-13, Japan \\ Yukiyasu Kobayashi \\ National Astronomical Observatory, Mitaka, Tokyo, 181, Japan
}

\section{Introduction}

Since molecular gas fuels AGNs and molecular clouds form stars, understanding of molecular gas dynamics is a key to the understanding of active phenomena (such as starbursts and AGNs) in galactic nuclei. To study gas dynamics in weakly barred galaxies, we made CO interferometry (to trace gas) and NIR imaging (to trace stars) toward two nearby SAB galaxies M100 and M94. Each galaxy has a small stellar nuclear bar and also has an outer bar or oval distortion, thus suitable for the study of gas dynamics in a barred gravitational potential. Observations were made using Nobeyama Millimeter Array (NMA) and the IRcamera PICNIC installed at the ISAS $1.3 \mathrm{~m}$ telescope.

\section{2. $\mathrm{M} 100(\mathrm{NGC} 4321)$}

Figure 1a shows $2.5^{\prime \prime}(210 \mathrm{pc} ; \mathrm{D}=17.1 \mathrm{Mpc})$ resolution CO map of M100. In the central $5 \mathrm{kpc}$, two-armed molecular spirals start at the ends of the nuclear stellar bar seen in our NIR images (see also Knapen et al. in this conference). There is a concentration of $\mathrm{CO}$ emission at the center, and distortions of velocity field at the arms (Figure 1b). These gas structures are successfully modeled using resonance orbits and self-gravity of gas. Figure 1c shows analytically calculated gas orbits (Wada 1994) in a barred potential, which explains the observed molecular arms.

\footnotetext{
${ }^{1}$ Department of Astronomy, University of Tokyo, Tokyo, 113, Japan
} 


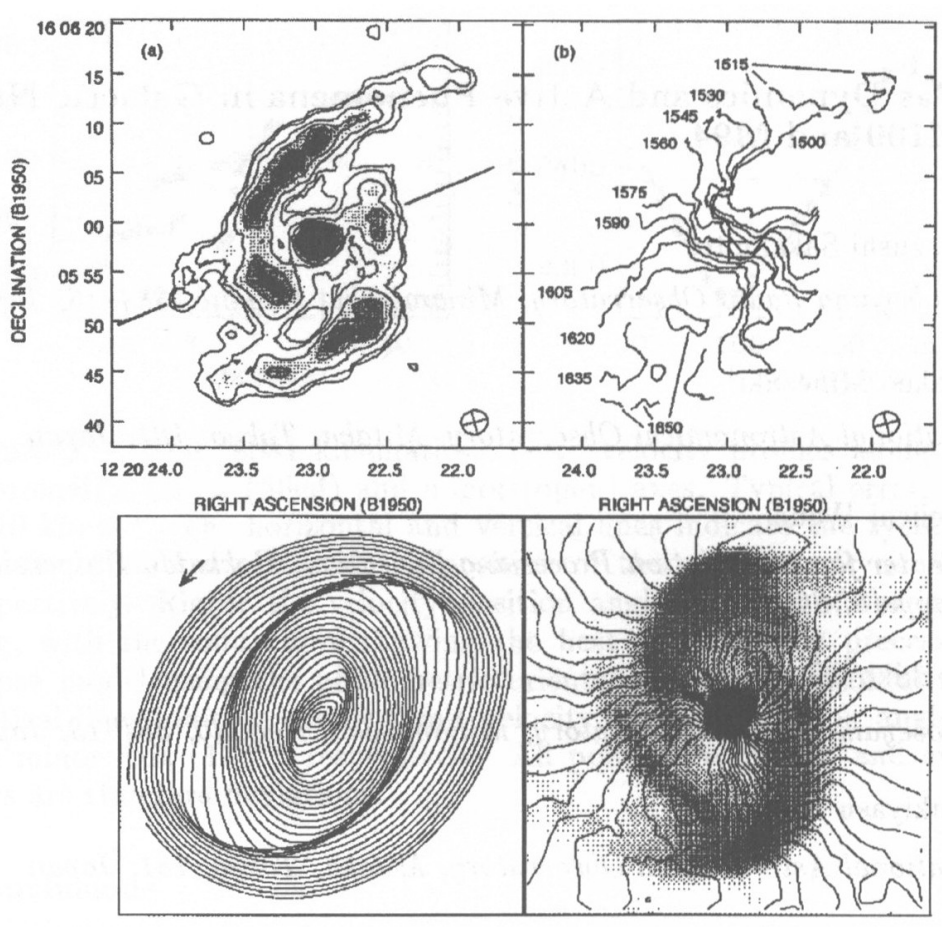

Figure 1. $\quad \mathrm{CO}(1-0)$ integrated intensity map (a), and isovelocity map (b) of M100. Analytically derived gas orbits (c), and numerically simulated gas distribution and velocity field (d).

There are two pairs of orbit-crowding spiral arms; the inner leading pair is at the inner inner Lindblad resonance (IILR) and the outer trailing pair is at the outer inner Lindblad resonance (OILR), and the observed CO arms corresponds to the OILR arms. Figure 1d shows more detailed numerical simulations of the gas distribution and velocity field in a barred potential; the OILR trailing spiral arms and distortions of isovelocity contours at the arms are very similar to the observations. These similarities strongly suggest the presence of OILR at the molecular arms. At the center of Figure 1d, there is a concentration of gas instead of the IILR spiral arms in Figure 1c. In the numerical simulation, the central gas once piled up at the IILR fragmented to form clumps due to selfgravitational instability, and spiraled into the center due to mutual collisions and subtraction of angular momentum by the bar (Wada \& Habe 1992). The dynamical friction between stars and gas clumps may also help the infall. The observed gas mass at the central peak is above the critical mass to cause the selfgravitational instability, thus the infall of molecular gas from the IILR may be a cause of the central gas concentration in M100 (for more details see Sakamoto et al. 1995.)

There are $\mathrm{H} \alpha$ hot-spots along the molecular spiral arms (Knapen et al. 1995), which may be triggered by the frequent cloud collisions, shocks, and 


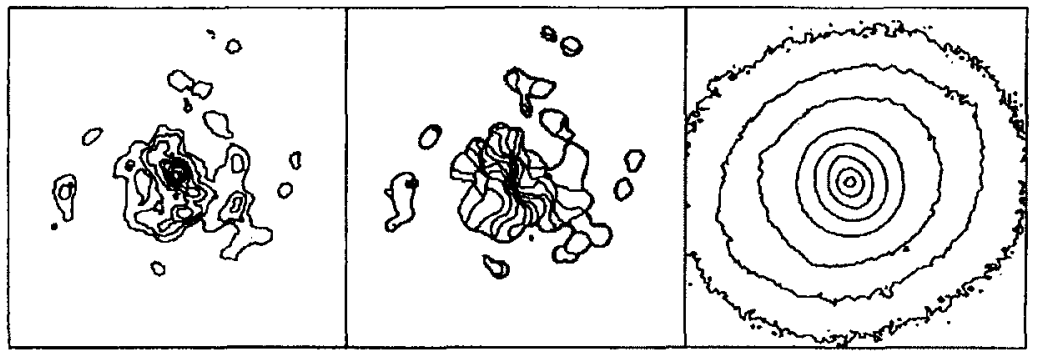

Figure 2. $\quad \mathrm{CO}(1-0)$ integrated intensity map (a), isovelocity map (b), and $K^{\prime}$ band image (c) of M94 in the central $2^{\prime}=3.6 \mathrm{kpc}$.

gravitational instability at the arms. As for the nuclear activity, M100 does not have a powerful AGN. Compared to the Seyfert 2 galaxy NGC 1068, which has similar nuclear gas and stellar bar structure, M100 has 3 times larger mass of molecular gas in the central $200 \mathrm{pc}$, but emits $10^{3}$ times less power of $20 \mathrm{~cm}$ emission from the nucleus, indicating that the bar-driven gas accumulation to $\sim 100$ pc radius is not sufficient for the onset of AGN activities.

\section{M94 (NGC 4736)}

There is a nuclear stellar bar at the center of M94 (Fig. 2c). In this region, the CO maps in Figure 2 show a nuclear gas concentration, gas associated with the bar, gas arcs locating at the ends of the nuclear bar, gas spiral arms, and large non-circular motions. The overall gas morphology suggests that the molecular gas in the central region is strongly perturbed by the nuclear bar. Since the $50^{\prime \prime}$ radius $\mathrm{H} \alpha$ ring of M94 is successfully modeled with a weak oval distortion of the outer disk (van Driel et al. this conference), two barred structures in M94 dominate the gas dynamics at the outer and inner parts separately. The relation between the two barred structures is not clear at present, but one interesting possibility is that they are rotating independently (Friedli this conference). Although the central region of M94 appears to be in post-starburst phase (Walker et al. 1988), the map does not show depletion of molecular gas at the center. In spite of the concentration of gas at the nucleus, M94 does not have a powerful AGN (classified as Liner in the literature), which is a case similar to M100.

\section{References}

Knapen, J. H., Beckman, J. E., Shlosman, I., Peletier, R. F., Heller, C. H., \& de Jong, R. S. 1995, ApJ, 443, L73

Sakamoto, K., Okumura, S., Minezaki, T., Kobayashi, Y., \& Wada, K. 1995, AJ, 110,2075

Wada, K. 1994, PASJ, 46, 165

Wada, K. \& Habe, A. 1992, MNRAS, 258, 82

Walker, C. E., Lebofsky, M. J., \& Rieke, G. H. 1988, ApJ, 325, 687 